\title{
The Effects of a Magnetic Field on the Crystallization of a Fluorozirconate Glass
}

Dennis S. Tucker and Michael R. Lapointe

Marshall Space Flight Center, MSFC, Alabama 35812

Zhiyong Jia

MINT Center, University of Alabama, Tuscaloosa, Alabama 35487

An axial magnetic field of $0.1 \mathrm{~T}$ was applied to $\mathrm{ZrF}_{4}-\mathrm{BaF}_{2}-\mathrm{LaF}_{3}-\mathrm{AlF}_{3}-\mathrm{NaF}$ (ZBLAN)

fibers during heating to the glass crystallization temperature. Scanning electron microscopy and $\mathrm{x}$-ray diffraction were used to identify crystal phases. It was shown that fibers exposed to the magnetic field did not crystallize while fibers not exposed to the field did crystallize. A hypothesis based on magnetic work was proposed to explain the results and tested by measuring the magnetic susceptibilities of the glass and crystal. 
Fluorozirconate glasses have been of interest to researchers for over 25 years. One class that has consistently shown the most promise is $\mathrm{ZrF}_{4}-\mathrm{BaF}_{2}-\mathrm{LaF}_{3}-\mathrm{AlF}_{3}-\mathrm{NaF}$ (ZBLAN) glass. ${ }^{1}$ ZBLAN fiber optics are currently used in a number of applications, such as fiber amplifiers, lasers for cutting, drilling and surgery and they show promise in applications, such as nuclear radiation resistant lengths and non-linear applications. ${ }^{2}$ Intrinsic and extrinsic processes limit light propagation at low powers in ZBLAN. ${ }^{3}$ Intrinsic processes include band-gap absorption, Rayleigh scatter and multiphonon absorption. Extrinsic processes include impurities, such as rare-earth and metal ions, and crystallites formed during preform preparation and fiber drawing. The theoretical loss coefficient for ZBLAN is $0.001 \mathrm{~dB} / \mathrm{km}$ at two micrometers. The achievement of this lower limit is hindered by both intrinsic and extrinsic processes. Varma et. al. ${ }^{4}$ stated that they felt the devitrification is due to a narrow working range and low viscosity at the drawing temperature. A low viscosity increases the likelihood of crystallization due to ionic diffusion..$^{5}$

Magnetic fields have been used to affect flows in conducting fluids. Crystals grown from melts are improved by using a uniform ${ }^{6,7,8}$ and rotating magnetic fields. ${ }^{10}$ Convective heat and mass transport in semiconductor melts with large electrical conductivities can be controlled by magnetic fields. The effects of DC and AC fields are different. A DC field tends to produce a body force which opposes or damps the buoyant convection, while $\mathrm{AC}$ fields drive melt motions. ${ }^{11}$ The effects of magnetic fields on $\mathrm{g}$ jitter driven flow under microgravity have also been investigated, ${ }^{12}$ as well as the effects of a magnetic field on the crystallization of inorganic salts. The reported results vary for 
magnetic field effects on inorganic salt crystallization; it has been found that calcium carbonate crystallization decreases under a magnetic field ${ }^{13}$, while soluble diamagnetic salts of weak acids were shown to have accelerated crystallization. ${ }^{14,15}$ In addition, Wang et. al. ${ }^{16}$ were able to retard crystallization in a bulk amorphous alloy using a high magnetic field (10T). N. Toyohara et. al. ${ }^{17}$ prepared Bi-based superconducting glassceramics in a $10 \mathrm{~T}$ field. They found that the superconducting properties were enhanced when the crystals with a plate-like shape tend to stack to the direction of the magnetic field. Also, N. Toyohara et. al. ${ }^{18}$, found that the c-axis orientation and second-harmonic intensity of $\mathrm{Ba}_{2} \mathrm{TiGe}_{2} \mathrm{O}_{8}$ crystals were enhanced in samples fabricated with a $10 \mathrm{~T}$ magnetic field perpendicular to the sample surface.

The purpose of this study was to determine if the crystallization in ZBLAN glass would be suppressed in a magnetic field. An axial field was chosen based on theoretical work by Chandrasekhar ${ }^{19}$, which shows gravitational perturbations in a liquid jet can be stabilized by an axial magnetic field.

A solenoid electromagnet with a field strength of $0.1 \mathrm{~T}$ was used for this investigation. The magnetic field direction could be reversed by changing the polarity on the power supply. A furnace capable of $400^{\circ} \mathrm{C}$ was constructed to hold the ZBLAN glass optical fibers. The furnace was mounted in the center of the electromagnet, providing accurate fiber alignment with the solenoid axis. The axial magnetic field was uniform $(+/-2 \%)$ over the full length of the ZBLAN fiber sample. A photograph of the test system is shown in figure 1. Nine fibers were used in this study (provided by Infrared Fiber Systems Inc., Silver Springs, Md). These fibers were $50 \mathrm{~mm}$ in length and 300 micrometers in diameter. A polymer buffer coating was chemically stripped. All fibers 
were encapsulated in fused quartz ampoules, and backfilled with helium to protect the samples from water contamination and to aid in heat transfer. Three samples were heated to the crystallization temperature $\left(345^{\circ} \mathrm{C}\right)$ for 45 seconds with the magnetic field vector parallel to the gravity vector and three samples were heated in the same manner with the magnetic field vector anti-parallel to the gravity vector. The final three samples were heated in the same manner but without a magnetic field. The heating time was limited by the resistance heating of the un-cooled electromagnetic coils. After processing the samples were observed with a stereo-optical microscope and a scanning electron microscope (SEM). X-ray diffraction was used to identify the crystal type.

The three control samples exhibited "frosting" representative of crystallization when viewed under optical microscopy. Further examination under SEM showed crystals with the appearance as in figure 2. The crystal structure was identified as $\mathrm{BaZrF}_{6}$, the most common crystal type for ZBLAN. The fibers heated in the magnetic field did not show evidence of crystallization under optical microscopy or SEM. An example of a fiber heated with the magnetic field vector anti-parallel to the gravity vector is shown in figure 3. Figure 4 is an example of a fiber heated with the magnetic field vector parallel to the gravity vector. Note that there are irregularities in the surface of this fiber. This was also true for the other two fibers tested in this manner.

It was hypothesized that the mechanism for suppression of crystallization was the permeability difference between the amorphous glass and the crystallites. Assuming the formation of spherical nuclei in solids the change in free energy without a magnetic field consists of three parts: bulk free energy, strain energy and surface energy ${ }^{16}$

$$
\Delta G=\frac{4}{3} \pi r^{3}\left(\Delta G_{\nu}+\varepsilon\right)+4 \pi r^{2} \sigma
$$


where $\mathrm{r}$ is the radius of the nucleus, $\Delta G_{v}$ and $\varepsilon$ is the bulk free energy decrease and strain energy increase per unit volume, respectively, and $\sigma$ is the interfacial energy increase per unit area of interface between a nucleus and matrix. The strain energy is usually negligible since the supercooled phase can relax any misfit induced strains.

When a magnetic field is applied to a material, the work induced by the magnetic field can be expressed as ${ }^{16}$

$G=\frac{1}{2} \int_{v} B H d v=\frac{1}{2} \int_{v} \mu H^{2} d v=\frac{1}{2} \mu H^{2} \frac{4}{3} \pi r^{3}$

where, $\mu$ is the magnetic permeability, and $H$ is the intensity of the applied magnetic field. As phase transformations occur, new phases precipitate from the matrix and the permeability of the sample changes from $\mu_{1}$ to $\mu_{2}$. Therefore, the contribution to phase transformation by the free energy change under a magnetic field is

$$
\Delta G_{M}=\frac{1}{2} H^{2}\left(\mu_{2}-\mu_{1}\right) \frac{4}{3} \pi r^{3}
$$

Here $\mu_{1}$ is the permeability of the parent phase (glass) and $\mu_{2}$ is the permeability of the new crystalline phase. In all, the total free energy change of a material under a magnetic field is given by

$$
\Delta G=\frac{4}{3} \pi r^{3}\left[\Delta G_{V}-\frac{1}{2} H^{2}\left(\mu_{2}-\mu_{1}\right)\right]+4 \pi r^{2} \sigma
$$

Then, the free energy required for the formation of a critical nucleus $\left(\Delta G_{C}\right)$ obtained by setting $d \Delta G / d r=0$ is

$$
\Delta G_{c}=\frac{16 \pi \sigma^{3}}{3\left[\left\langle\Delta G_{v}\right\rangle+\frac{1}{2} H^{2}\left(\mu_{2}-\mu_{1}\right)\right]^{2}}
$$


where $\left\langle G_{V}\right\rangle$ is defined as the absolute value of the volume free energy.

From equation (5), it can be seen that the effect of a magnetic field yields two measurable variables: The intensity of the applied magnetic field and the permeability difference.

To test this hypothesis that permeability may play a role in suppressing crystallization, the magnetic susceptibility of ZBLAN glass and crystallized ZBLAN were measured. A $4 \mathrm{~mm}$ thick by $11 \mathrm{~mm}$ diameter sample of glass was crystallized fully by heating to $345^{\circ} \mathrm{C}$ for 6 hours.

The magnetic susceptibility of the samples was measured using a vibrating sample magnetometer (Digital Measurement System Model 880 VSM) with a maximum applied field strength of $13 \mathrm{kOe}$. The VSM is calibrated using a Ni standard sample with $6.35 \mathrm{~mm}$ diameter and 50 micrometers thickness, which has a saturation magnetic moment of $0.718 \pm 0.011 \mathrm{emu}$ at $20^{\circ} \mathrm{C}$. The background moment from a quartz sample holder and substrate was also measured and automatically subtracted during measurement. The relative values of susceptibility can be compared to within $1 \%$ and the absolute accuracies to within $5 \%$. These are only estimates, based upon experience of the experimenter, since only one crystallized sample and one glass sample were measured. More definitive values could be arrived at by doing repeated measurements on many different samples. The dimensionless volume magnetic susceptibility, represented by the symbol $\chi_{v}$, is defined by $M=\chi_{\nu} H$

where $M$ is the magnetization of the material (magnetic dipole moment per unit volume), measured in emu, and $H$ is the applied magnetic field, measured in Oe. Thus, the magnetic susceptibility is defined by the ratio of $M / H$. 
The susceptibilities were converted to S.I. units and the permeability was calculated using:

$\mu=\mu_{0}\left(1+\chi_{V}\right)$

where $\mu_{0}=4 \pi \times 10^{-7} N / A^{2}$ is the permeability of free space.

There is no significant numerical difference between the calculated permeability values, which eliminates a difference in permeability between the glass and crystal as being the mechanism of suppressed crystal growth.

The viscosity of the melt is a directly measurable kinetic parameter used in nucleation and growth equations. According to Turnbull ${ }^{20}$, the nucleation rate, $I$, and the growth rate, $\mathrm{U}$, are both inversely proportional to viscosity. It has been reported that in semiconductor melts, the flow velocity is attenuated and viscosity increases above a critical magnetic field strength $(\sim 0.1 \mathrm{~T})^{21}$ A similar mechanism may be at work here. At the crystallization temperature, the viscosity of ZBLAN is $\leq 10^{4}$ poise, which would allow ionic diffusion. An increase in the viscosity would then slow diffusion and crystallization would be slowed. We plan to pursue this hypothesis utilizing a superconducting magnet with a vertically aligned axial field. The magnet has a high temperature furnace $\left(1000^{\circ} \mathrm{C}\right)$ suitable for these studies, and experiments are planned to measure the viscosity of ZBLAN glass as a function of temperature and magnetic field.

This work was supported by the Marshall Space Flight Center Technology Transfer Office. The authors would like to thank Mr. Guy Smith of Tecmasters, Huntsville, Alabama and Professor J.W. Harrell of the University of Alabama for their valued input to this project. 
1. L. Boehm, K.H. Chung, S.N. Crichton and C.T. Moynihan, Infrared Optical Materials and and Fibers V, SPIE 843, 10, 1987.

2. D.C. Tran, G.H. Siegel and B. Bendow, J. Lightwave Tech., LT-2, 5, 121, 1984.

3. N.P. Bansal, A.J. Bruce, R.J. Doremus and C.T. Moynihan, Mat. Res. Bull., 19, 522, 1984.

4. S. Varma, S.E. Prasad, and I Murley, Proc. Spacebound, 91, 248, 1991.

5. J.S. Sanhera, L.E. Busse, I.D. Aggarwal and C. Rapp, in Infrared Fiber Optics eds, J.S. Sanghera and I.D. Aggerwal, 75, 1998.

6. D.T.J. Hurle, Handbook of Crystal Growth, North-Holland, Amsterdam, 1994.

7. V. Galindo, G.Gerbeth, W. von Ammon, E. Tomzig and J. Virbulis, Energy Convers. Manage., 43, 309, 2002.

8. A.I. Fedoseyev, E.J. Kansa, C. Marin, A.G. Ostrogorsky, Proceedings of the $8^{\text {th }}$ ISCFD, ZARM Bremen, 1999.

9. P.J. Prescott and F.P. Incropera, Trans. ASME, 115, 202, 1993

10. O. Patzold, I. Grants, U. Wunderwald, K. Jenker, A. Croll and G. Gerbeth, J. Cryst. Growth, 245, 237, 2002.

11. J.S. Walker, Progress in Crystal Growth and Characterization, 195, 1999.

12. B. Pan, D-Y Shang, B.Q. Li and H.C. de Groh, Intern. J. of Heat and Mass Transfer, 45, 125, 2002.

13. H. E. Lundager Madsen, J. of Cryst. Growth, 267, 251, 2004. 
14. A.M.B. Freitas, F.J.G. Landgraf, J. Nyvlt and M. Giulietti, Cryst. Res. Technol., 34, $1239,1999$.

15. H.E. Lundager Madsen, J. of Cryst. Growth, 152, 94, 1995.

16. X. Wang, M. Qi and S. Yi, Scripta Mat., 51, 1047, 2004.

17. N. Toyohara, Y. Benino, T. fujiwara, S. Tanaka, K. Uematsu and T. Komatsu, Physica C, 420, 88, 2005.

18. N. Toyohara, Y. Benino, T. Fujiwara, S. Tanaka, K. Uematsu and T. Komatsu, J. Applied Phys., 99, 043515-1, 2006.

19. S. Chandrasekhar, Hydrodynamic and Hydromagnetic Stability, Dover Publications, N.Y. 1961

20. D. Turnbull, Contemp. Phys., 10, 473, 1969

21. Y. Miyazawa, Progress in Cryst. Growth and Charact., 261, Pergamon Press, Amsterdam, 1999. 
Fig. 1. Experimental setup showing electromagnet, temperature controller and power supply.

Fig. 2. Backscattered electron micrograph of ZBLAN fiber heated to crystallization temperature without magnetic field showing crystals.

Fig. 3. Backscattered electron micrograph of ZBLAN fiber heated to crystallization temperature with magnetic field vector anti-parallel to the gravity vector.

Fig. 4. Backscattered electron micrograph of ZBLAN fiber heated to crystallization temperature with magnetic field parallel to gravity vector. 


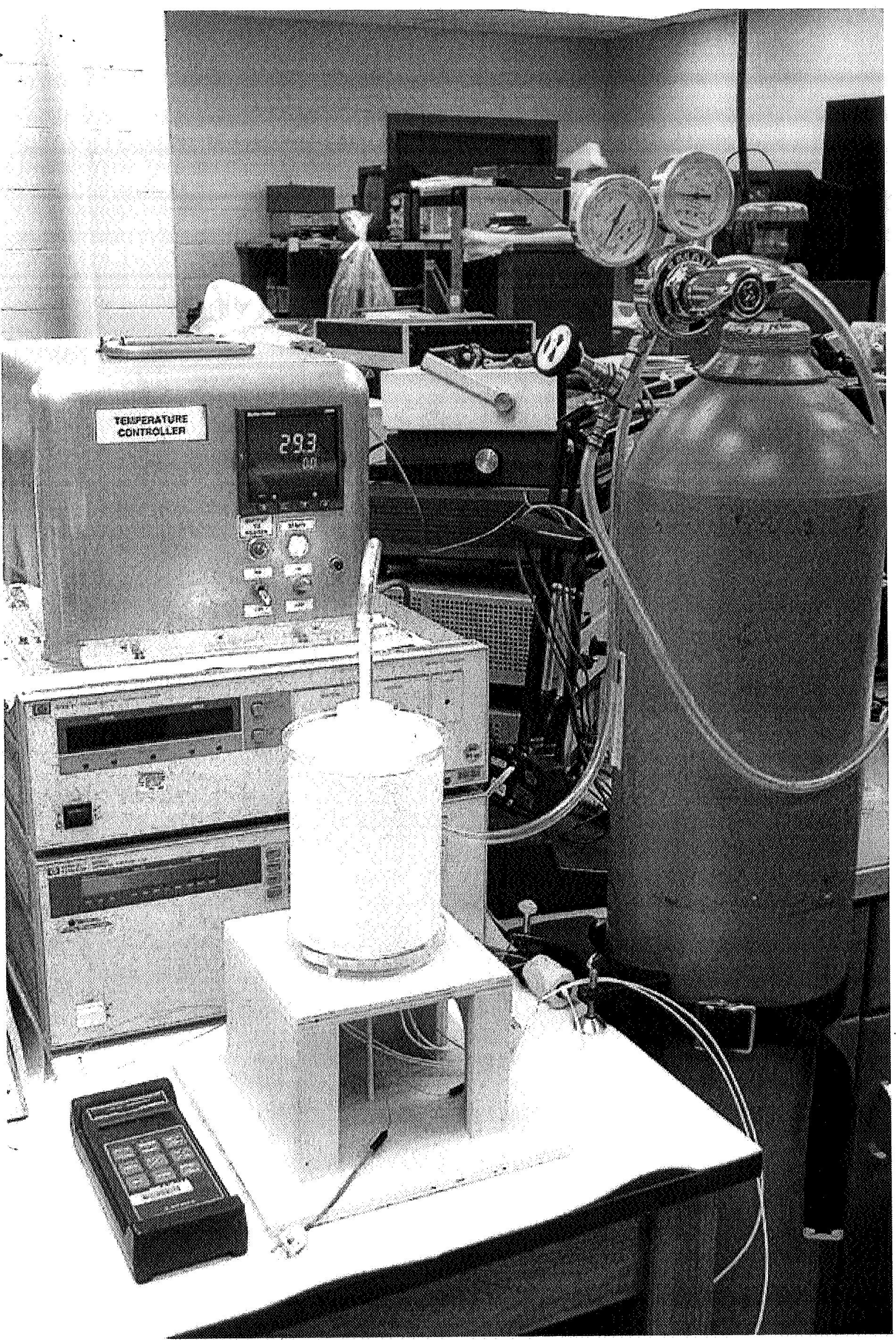




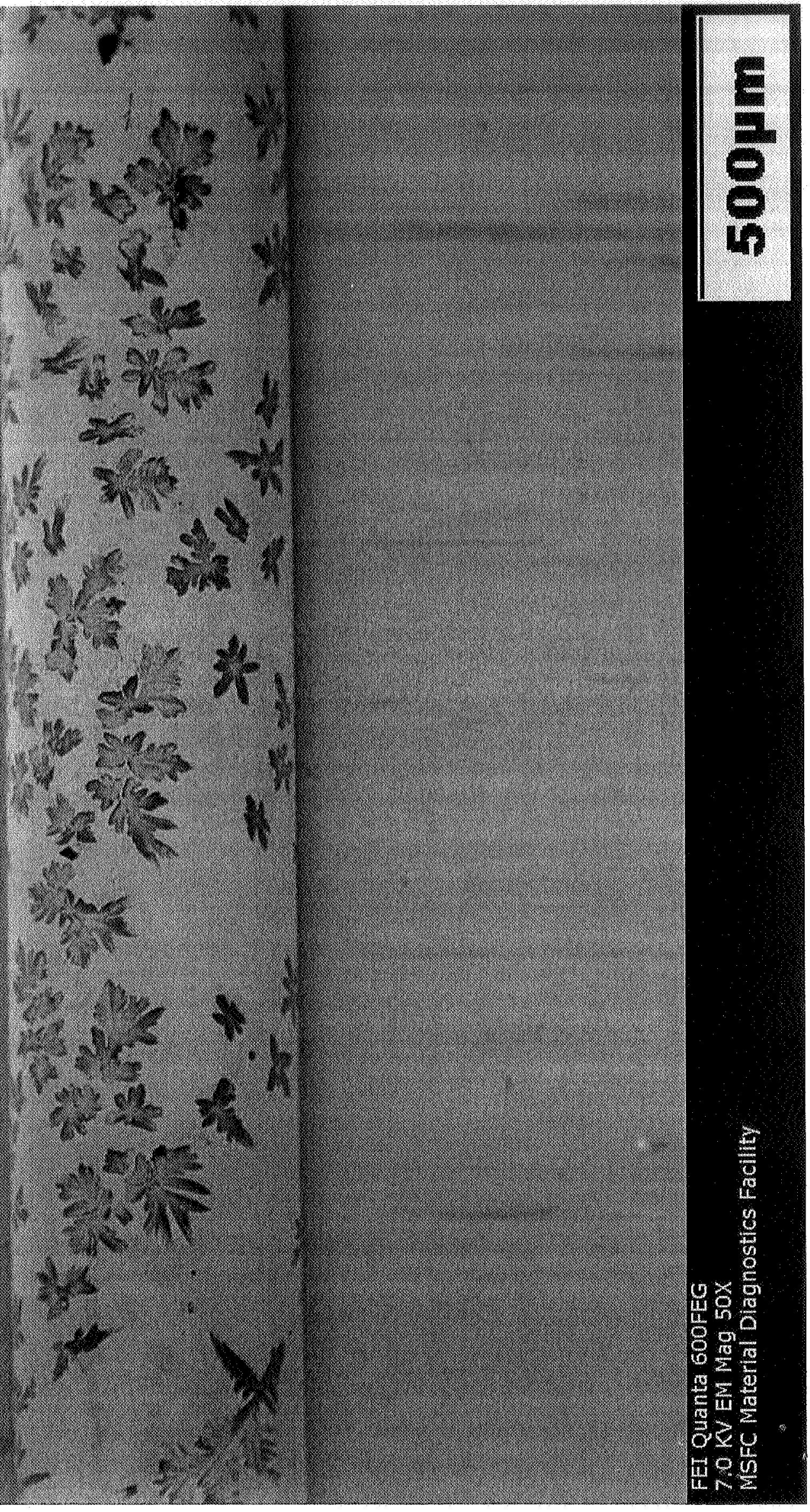




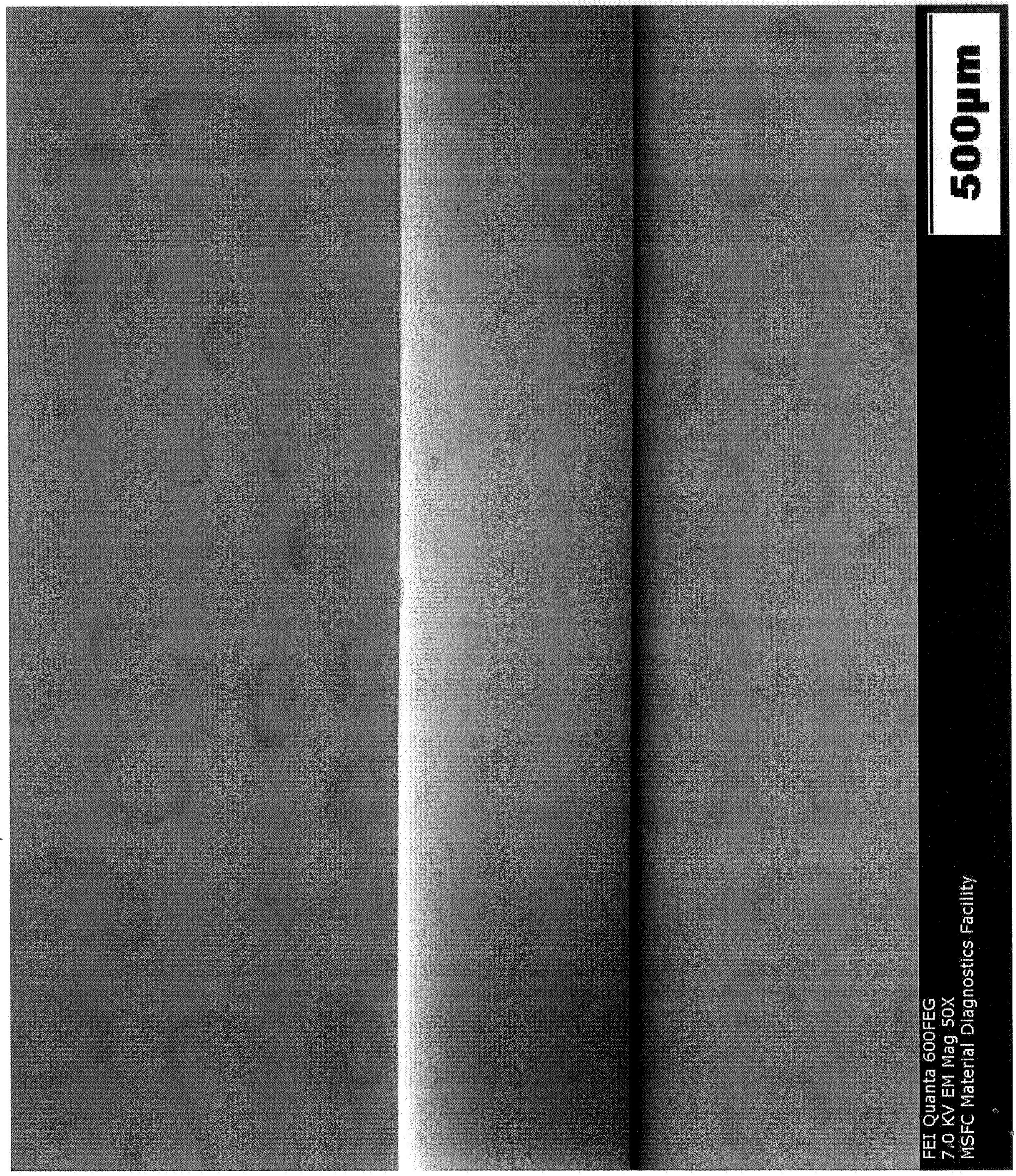




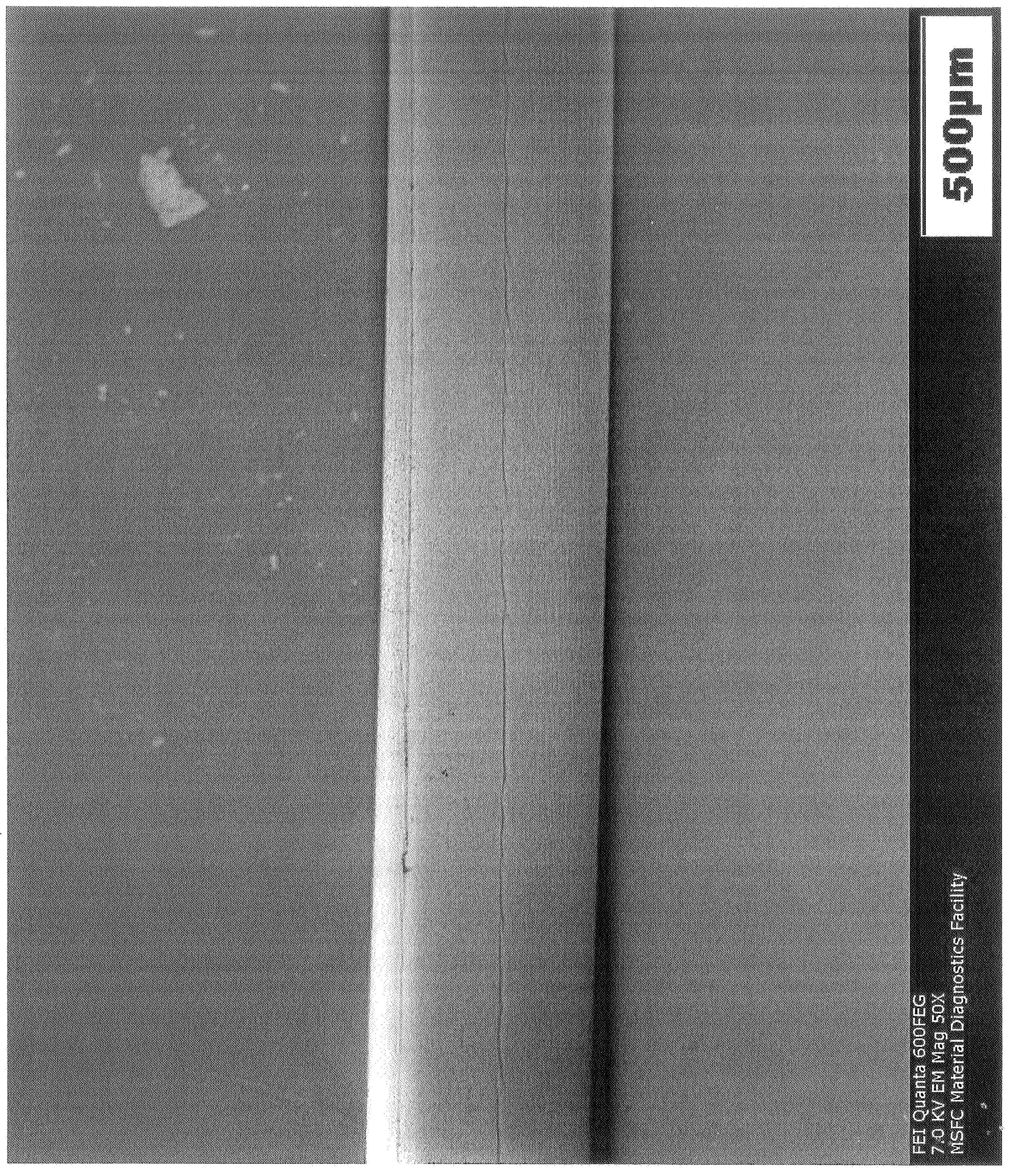

